\title{
Microduplications of 22q11.2 are frequently inherited and are associated with variable phenotypes
}

Zhishuo Ou, $M D^{1}$, Jonathan S. Berg, $M D, P h D^{1}$, Hagith Yonath, $M D^{1}$, Victoria B. Enciso, $M S^{2}$, David T. Miller, $M D, P h D^{3}$, Jonathan Picker, $M D^{3}$, Tiffanee Lenzi, $M D, P h D^{4}$, Catherine E. Keegan, $M D, P h D^{4}$, Vernon R. Sutton, $M D^{1,5}$, John Belmont, $M D, P h D^{1,5}$, A. Craig Chinault, PhD ${ }^{1}$, James R. Lupski, $M D, P h D^{1,5,6}$, Sau Wai Cheung, $P h D, M B A^{1}$, Elizabeth Roeder, $M D^{2}$, and Ankita Patel, $P h D^{1}$

\begin{abstract}
Purpose: Genomic rearrangements of chromosome 22q11.2, including the microdeletion associated with DiGeorge/velocardiofacial syndrome, are mediated by nonallelic homologous recombination between region-specific low-copy repeats. To date, only a small number of patients with 22q11.2 microduplication have been identified. Methods: We report the identification by array-comparative genomic hybridization of 14 individuals from eight families who harbor microduplications within the 22q11.2 region. Results: We have now observed a variety of microduplications, including the typical common $\sim 3-\mathrm{Mb}$ microduplication, $\sim 1.5-\mathrm{Mb}$ nested duplication, and smaller microduplications within and distal to the DiGeorge/velocardiofacial syndrome region, consistent with nonallelic homologous recombination using distinct low-copy repeats in the 22q11.2 DiGeorge/velocardiofacial syndrome region. These microduplications likely represent the predicted reciprocal rearrangements to the microdeletions characterized in the 22q11.2 region. The phenotypes seen in these individuals are generally mild and highly variable; familial transmission is frequently observed. Conclusions: These findings highlight the unbiased ability of array-comparative genomic hybridization to identify genomic imbalances and further define the molecular etiology and clinical phenotypes seen in microduplication 22q11.2 syndrome. Our findings also further support that the 22q11.2 region is highly dynamic with frequent rearrangements using alternative low-copy repeats as recombination substrates. Genet Med 2008:10(4):267-277.
\end{abstract}

Key Words: 22q11.2, microduplication, DiGeorge/velocardiofacial syndrome, low-copy repeats

Genomic disorders result from DNA rearrangements caused by nonallelic homologous recombination (NAHR) between region-specific low-copy repeats (LCRs) or nonhomologous end joining, leading to interstitial deletions, duplications, and inversions as well as unbalanced translocations. ${ }^{1,2}$ Chromosome 22q11.2 is a well-characterized genomic region that is gene-rich and contains multiple region-specific LCRs, designated LCR22s (LCR22-1 through LCR22-8) ${ }^{3}$ or alternately referred to by alphabetic designations. ${ }^{4}$ These LCRs are known to

\footnotetext{
${ }^{I}$ Department of Molecular and Human Genetics, Baylor College of Medicine, Houston, Texas; ${ }^{2}$ Department of Pediatrics, UTHSCSA, San Antonio, Texas; ${ }^{3}$ Division of Genetics and Department of Laboratory Medicine, Children's Hospital, Boston, Massachusetts; ${ }^{4}$ Department of Pediatrics, Division of Genetics, University of Michigan, Ann Arbor, Michigan; ${ }^{5}$ Texas Children's Hospital, Houston, Texas; and ${ }^{6}$ Department of Pediatrics, Baylor College of Medicine, Houston, Texas.

Sau Wai Cheung, PhD, MBA, Department of Molecular and Human Genetics, Baylor College of Medicine, One Baylor Plaza, NAB2015, Houston, TX 77030.E-mail: scheung@bcm.edu.

The first three authors contributed equally to this work.

Disclosure: The authors declare no conflict of interest.

Submitted for publication September 26, 2007.

Accepted for publication January 29, 2008.

DOI: $10.1097 / G I M .0 b 013 e 31816 b 64 c 2$
}

mediate genomic rearrangements in this region, ${ }^{5}$ resulting in genomic disorders such as DiGeorge/velocardiofacial syndrome (DGS/VCFS), ${ }^{6}$ cat eye syndrome, ${ }^{7} \operatorname{der}(22)$ syndrome, ${ }^{8}$ and the 22q11.2 microduplication syndrome. ${ }^{9}$

DGS/VCFS is the most frequently identified genomic disorder of the 22q11.2 region with an estimated frequency of 1 of 4000 live births. ${ }^{10}$ The clinical description of DGS/VCFS (MIM 188400,192340 ) includes the triad of cardiovascular malformations (particularly conotruncal abnormalities), hypocalcemia caused by hypoplasia of the parathyroid glands, immune dysfunction caused by thymic aplasia, facial dysmorphic features, and developmental delay with a highly variable phenotype. ${ }^{11,12}$ Testing of family members of affected individuals has led to the identification of individuals with very mild clinical manifestations, further expanding the phenotypic spectrum seen with this microdeletion. ${ }^{13}$ Most of the patients (80-90\%) with DGS/VCFS have a common $\sim 3-\mathrm{Mb}$ microdeletion of chromosome 22q11.2 between LCR22-2 and LCR22-4, whereas most of the remaining patients have a $\sim 1.5-\mathrm{Mb}$ nested deletion with breakpoints mapping between LCR22-2 and LCR22-3a. ${ }^{14}$ There have also been further reports of rare patients with atypical deletions within the region. ${ }^{15-22}$ 


\section{Ou et al.}

Based on the mechanism by which recurrent chromosome 22q11.2 microdeletions occur, it was postulated that individuals carrying chromosomes with the reciprocal microduplications should also exist. ${ }^{1}$ Because LCR22s mediate NAHR events that result in rearrangements of 22q11.2 owing to unequal crossovers during meiosis, deletion on one chromosome should result in duplication on the other. As expected, a proband and several additional family members carrying the 22q11.2 microduplication were identified. ${ }^{3}$ Additional case reports led to the further characterization of the duplication 22q11.2 syndrome as a new genomic disorder that is complementary to, but distinct from, the 22q11.2 deletion syndrome. ${ }^{9,23-25}$ Molecular analyses have revealed that individuals with 22q11.2 microduplication typically carry a $\sim 3-\mathrm{Mb}$ duplication that is the reciprocal of the common $\sim 3-\mathrm{Mb}$ deletion found in DGS/VCFS, although larger duplications of $\sim 4-\mathrm{Mb}$ and $\sim 6-\mathrm{Mb}$ that include the DGS/VCFS region were also reported. ${ }^{9}$ The phenotypes previously associated with microduplications of 22q11.2 include cardiovascular anomalies, velopharyngeal insufficiency with or without cleft palate, hearing loss, growth and developmental delay, learning disabilities, behavioral problems, and various dysmorphic features. Based on the current literature, the phenotypes in patients with microduplication of 22q11.2 may have partial overlap with DGS/ VCFS $9,23,24$; however, this degree of overlap is almost certainly the result of ascertainment bias, because the patients thus far reported to harbor 22q11.2 microduplications were generally selected on the basis of clinical features that prompted referral to rule out VCFS/DGS microdeletion. 9,25

Despite the frequency with which 22q11.2 microdeletion occurs, only a small number of patients with microduplication of this region have been described to date; this may be, in part, owing to a highly variable and mild phenotype such that many individuals with microduplications within 22q11.2 may not undergo testing. In addition, the technical limitations of testing by fluorescence in situ hybridization (FISH) may prevent the identification of many affected individuals in that duplications may only be visualized by FISH using interphase cells. Modern techniques such as array comparative genomic hybridization (aCGH) can now be used as a clinical diagnostic test for a variety of genomic disorders ${ }^{26,27}$ and will likely lead to identification of much larger numbers of patients with microdeletions and microduplications. In our experience of approximately 7000 clinical aCGH cases, we have detected 59 patients with gains or losses within 22q11.2, including 19 individuals from 10 families who carry microduplications of various sizes within the DGS/VCFS region. Here, we present the clinical and molecular characterization of eight probands, including three probands with $\sim 3-\mathrm{Mb}$ microduplications consistent with reciprocal microduplication of the common DGS/VCFS microdeletion, two probands with $\sim 1.5-\mathrm{Mb}$ microduplications consistent with reciprocal microduplication of the proximal nested DGS/VCFS deletion, one proband with a $\sim 1$-Mb microduplication within the distal DGS/VCFS region, and two probands with microduplications distal to the region commonly deleted in DGS/VCFS. There is a high rate of familial transmission of 22q11.2 microduplications in this cohort, consistent with previous reports. The clinical phenotypes seen in these individuals are highly variable and relatively mild in comparison to those previously reported.

\section{MATERIALS AND METHODS}

Blood samples from patients referred to the Baylor cytogenetics laboratory for clinical aCGH analysis (chromosomal microarray [CMA]) were processed as described below. Informed consent approved by the Institutional Review Board for $\mathrm{Hu}-$ man Subject Research at Baylor College of Medicine and appropriate institutions was obtained for high-resolution oligonucleotide array analysis.

\section{Microarray constitution, hybridization, and analysis}

Microarrays containing 853 bacterial artificial chromosome (BAC) and P1-derived artificial chromosome clones designed to cover genomic regions of 75 known genomic disorders, all 41 subtelomeric regions, and 43 pericentromeric regions (BCM Medical Genetics Laboratories Website) were manufactured as described. ${ }^{28}$ Clones in the DGS/VCFS region were selected to flank LCR22s and have the ability to identify typical and atypical gains or losses. DNA labeling and hybridization were performed as previously described. ${ }^{26,29}$ Briefly, for each patient sample, two experiments were performed with reversal of the dye labels for the control and test samples, and the data from both dye-reversed hybridizations were integrated to determine inferences for each case. Quantitation data were subjected to normalization, and the dye-reversed data were combined to determine a single fold-change value for each clone. Inferences were made for all clones using these final combined data values. All analyses were performed on $\log _{2}$ ratios using code for the normalization and inference that was implemented in the $\mathrm{R}$ statistical programming language.

Whole Human Genome Oligo Microarray Kits and 44K (Agilent Technologies, Inc., Santa Clara, CA) were used to further refine the identified genomic gains and losses in selected patients. The procedures for DNA digestion, labeling, and hybridization were performed according to the manufacturer's instructions with some modifications. ${ }^{30}$

\section{Chromosome and FISH analyses}

Chromosome analysis was performed using standard protocols. Interphase FISH was performed using standard procedures with BACs labeled with Spectrum Orange or Spectrum Green (Abbot, Abbot Park, IL) by nick translation. Analysis of the genomic duplication was performed by visualizing twocolor FISH images showing three hybridization signals for the experimental probe and two signals for the control probe. At least 50 interphase nuclei were examined.

\section{RESULTS}

Genomic losses or gains within the 22q11.2 region were detected by aCGH in 59 individuals among approximately 7000 
samples referred for a variety of indications to undergo clinical testing over the course of several years. The samples were evaluated on several different versions of a targeted BAC array that included multiple clones covering the 22q11.2 region. Of these, 33 individuals have the common $\sim 3$-Mb VCFS/DGS microdeletion, one has the proximal nested $\sim 1.5-\mathrm{Mb}$ microdeletion, one has an atypical $\sim 4-\mathrm{Mb}$ deletion, and five harbor atypical microdeletions involving the region distal to the common VCFS/DGS interval. In addition, 10 individuals were found to carry $\sim 3-\mathrm{Mb}$ microduplications of the common VCFS/DGS region, and nine individuals were found to carry smaller atypical microduplications within or distal to the 22q11.2 region. Two individuals with the $\sim 3-\mathrm{Mb}$ duplication also had apparently balanced de novo translocations, and therefore, were excluded from this study. All of the microduplications detected by aCGH were independently confirmed by interphase FISH analysis.

To further characterize the phenotypic spectrum seen in patients with microduplications of 22q11.2, we obtained clinical information for eight unrelated probands. Of these eight individuals, three carried microduplications of the common $\sim 3-\mathrm{Mb}$ region most frequently deleted in DGS/VCFS. Two probands were found to have $\sim 1.5-\mathrm{Mb}$ duplications of the region previously reported as a "proximal nested" DGS/VCFS deletion. Three probands harbored atypical duplications ranging from $\sim 1$ to $\sim 2-\mathrm{Mb}$ in size. The molecular characterization of these duplications and the phenotypes of the patients are described below. Subsequent analyses of family members revealed six additional individuals from four families who harbor duplications (two fathers, two mothers, and two sisters). In all, clinical information was available for 12 individuals consisting of 8 probands with age at diagnosis ranging from 2 months to 10 years, and 4 relatives (summarized in Tables 1 and 2).

\section{Molecular characterization and phenotypes of patients with common $\sim 3-$ Mb microduplications}

Identification of the common $\sim 3-M b 22 q 11.2$ microduplication, LCR22-2 through LCR22-4

Patients 1-3 were found to have gain of copy number in the 22q11.2 region detected with BAC clones RP11-186O8, RP11316L10, and RP11-165F18 but normal copy number with flanking clones RP11-319F4 (proximal) and RP11-36N5 (distal; Fig. 1, A). These data are most consistent with $\sim 3-\mathrm{Mb}$ microduplications in which the proximal breakpoint lies within LCR22-2 and the distal breakpoint lies within LCR22-4. High-resolution genome analysis in Patient 1 using the Agilent 44K oligonucleotide array confirmed and further refined the suspected breakpoints (Fig. 1, B and C). Interphase FISH analysis independently characterized the gain of copy number as a duplication in each case (Fig. $1, \mathrm{D})$. Thus, the duplicated region likely represents the reciprocal recombination product to the common deletion in DGS/VCFS.

Phenotypes of patients with the common $\sim 3-\mathrm{Mb} 22 q 11.2$ microduplication, LCR22-2 through LCR22-4

All of the three probands with the common $\sim 3-\mathrm{Mb}$ microduplication were noted to have some degree of global develop-
Table 1

Clinical findings in patients with $\sim 3 \mathrm{Mb}$ and proximal "nested" $\sim 1.5 \mathrm{Mb}$ microduplications

$\sim 1.5 \mathrm{Mb}$

"nested"(LCR22-2

through 22-3a)

\begin{tabular}{|c|c|c|c|c|c|c|c|c|c|}
\hline \multirow[b]{2}{*}{ Patient } & & \\
\hline & 1 & $1 \mathrm{~S}$ & $1 \mathrm{M}$ & 2 & 3 & 4 & $4 \mathrm{~S}$ & $4 \mathrm{~F}$ & 5 \\
\hline $\begin{array}{l}\text { Age at } \\
\text { diagnosis }\end{array}$ & $3 \mathrm{yr}$ & $4-1 / 2 \mathrm{yr}$ & $29 \mathrm{yr}$ & $5 \mathrm{yr}$ & $10 \mathrm{mo}$ & $3 \mathrm{yr}$ & $2 \mathrm{yr}$ & $20 \mathrm{yr}$ & $4 \mathrm{yr}$ \\
\hline Sex & M & $\mathrm{F}$ & F & M & $\mathrm{F}$ & $\mathrm{F}$ & $\mathrm{F}$ & M & M \\
\hline $\begin{array}{l}\text { Developmental } \\
\text { delay }\end{array}$ & & & + & + & + & & + & & \\
\hline $\begin{array}{l}\text { Isolated speech } \\
\text { delay }\end{array}$ & + & + & & & & + & & + & + \\
\hline $\begin{array}{l}\text { Hypernasal } \\
\text { speech }\end{array}$ & & + & & + & & + & & + & \\
\hline $\begin{array}{l}\text { Hearing } \\
\text { impairment }\end{array}$ & + & & & + & + & & & & \\
\hline $\begin{array}{l}\text { Failure to } \\
\text { thrive }\end{array}$ & & + & & & & + & + & & \\
\hline $\begin{array}{l}\text { Abnormal } \\
\text { behavior }\end{array}$ & + & & & + & & & & & + \\
\hline ADHD & & & & + & & & & & \\
\hline
\end{tabular}

mental delay or speech delay in combination with variable dysmorphic features. Other features noted in more than one individual were hypernasal speech, abnormal behavior, and hearing impairment. None of the individuals were reported to have significant cardiovascular malformations or other significant birth defects. Clinical summaries of each individual are summarized in Tables 1 and 2.

Patient 1 is a 3 -year-old male child with mild speech delay, craniosynostosis, mild dysmorphic features, and normal growth parameters (Fig. 2, A). He has no cardiac or renal abnormalities, but auditory testing revealed mild bilateral conductive hearing loss. The patient's mother (referred to as patient $1 \mathrm{M}$ ) and sister (referred to as patient 1S) were both found to harbor the duplication. Patient $1 \mathrm{M}$ has mild dysmorphic features and required special education in high school, eventually completing 11 th grade. Patient $1 S$ is a $4 \frac{1}{2}-$-year-old female child with a history of failure to thrive, mild speech delay with hypernasal speech, and mild dysmorphic features. Patient 2 is a 5-year-old male child with global developmental delay, hypernasal speech, attention deficit hyperactivity disorder, frontal bossing and midface hypoplasia, difficulty with coordination and balance, and hypocalcemia. Parental samples were not available for testing. Patient 3 is a 10 -month-old female child with mixed conductive and sensorineural hearing loss, gross motor delay, macrocephaly and mild dysmorphic features. DNA sequencing of the GJB2 (Connexin-26) gene revealed no sequence changes associated with deafness. A head computed tomography scan revealed normally formed cochlea and semicircular canals but bilateral opacification of the middle ear. Parental samples were unavailable for evaluation. 
Table 2

Dysmorphic features identified in patients with $\sim 3 \mathrm{Mb}$ and proximal "nested" $\sim 1.5 \mathrm{Mb}$ microduplications

\begin{tabular}{|c|c|c|c|c|c|c|c|c|}
\hline \multirow[b]{2}{*}{ Patient } & \multicolumn{5}{|c|}{ 3 Mb "nested" } & \multicolumn{3}{|c|}{$\sim 1.5 \mathrm{Mb}$ "nested" } \\
\hline & 1 & $1 \mathrm{~S}$ & $1 \mathrm{M}$ & 2 & 3 & 4 & $4 \mathrm{~S}$ & $4 \mathrm{~F}$ \\
\hline
\end{tabular}

Head

Microcephaly

Abnormal suture

Brachycephaly

Hair

\section{Abnormal hair whorl}

Hirsutism

Eyes

\section{Upslanting \\ Downslanting \\ Epicanthal folds \\ Strabismus}

Midface hypoplasia

Ears

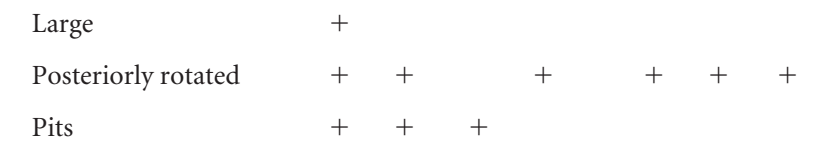

Nose

Broad or flat bridge

Depressed tip

Short columella

Prominent

Mouth

Smooth philtrum

Micrognathia

Bifid uvula

Other

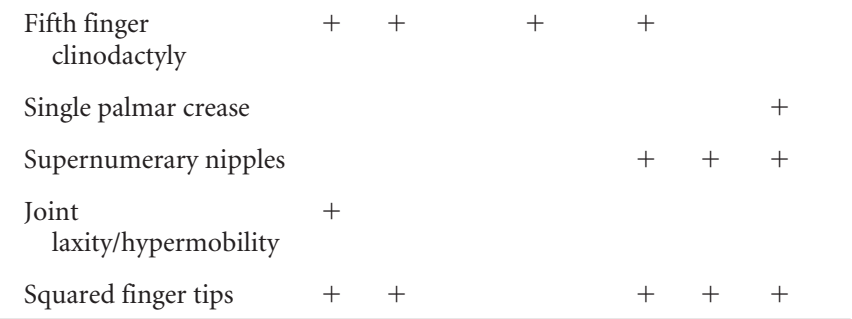

\section{Molecular characterization and phenotypes of patients with atypical $\sim 1.5-\mathrm{Mb}$ proximal nested microduplications}

Identification of the proximal nested $\sim 1.5-\mathrm{Mb} 22 q 11.2$ microduplication, LCR22-2 through LCR22-3a

Patients 4 and 5 were found to have gain of copy number with CMA clones RP11-186O8 and RP11-316L10 but normal copy number with flanking clones RP11-319F4 (proximal) and RP11-165F18 (distal; Fig. 3). High-resolution genomic analysis using the Agilent $44 \mathrm{~K}$ oligonucleotide array demonstrated that the duplication in Patient 4 extends between probes mapping to nucleotides $17,276,972 \mathrm{bp}$ and $18,691,704$ bp based on build hg18 (Fig. 3, B and C). Interphase FISH analysis independently characterized the gain of copy number as a duplication in both cases (Fig. 3, D). These data are consistent with a $\sim 1.5-\mathrm{Mb}$ microduplication in which the proximal breakpoints lie within LCR22-2 and the distal breakpoints lie within LCR22-3a, and thus, the duplicated region seems to be the reciprocal recombination product to the proximal nested $\sim 1.5-\mathrm{Mb}$ DGS/VCFS deletion.

Phenotypes of patients with the proximal nested $\sim 1.5-\mathrm{Mb}$

22q11.2 microduplication, LCR22-2 through LCR22-3a

All of the individuals with the proximal nested $\sim 1.5-\mathrm{Mb}$ microduplication were reported to have some degree of developmental delay, predominantly isolated speech delay, in combination with variable dysmorphic features (Fig. 2, B). Other features noted in more than one individual were hypernasal speech and failure to thrive. None of the individuals were reported to have significant cardiovascular malformations or other significant birth defects. Clinical findings in each individual are summarized below and in Tables 1 and 2 .

Patient 4 is a 3 -year-old female child with failure to thrive, speech delay, hypernasal speech, dysmorphic features, and splenomegaly. On testing of family members it was found that both her father (referred to as Patient 4F) and sister (referred to as Patient $4 \mathrm{~S}$ ) also harbor the duplication. Patient $4 \mathrm{~F}$ has a history of a heart murmur that resolved spontaneously, speech delay, hypernasal speech, and required special education while in grade school and high school. He has short stature, microcephaly, and mild dysmorphic features. Patient $4 \mathrm{~S}$ is a 2-yearold with failure to thrive, mild developmental delay, and dysmorphic features. Patient 5 is a 4 -year-old male child with speech delay, hyperactivity, and mild dysmorphic features. $\mathrm{Pa}$ rental samples were found to have normal FISH analysis using a commercial probe (TUPLE1 [Vysis] in the critical DGS/ VCFS) at an outside laboratory, indicating that the duplication was de novo.

\section{Molecular characterization and phenotypes of patients with unique microduplications}

Patients 6-8 were found to have gain of copy number with single CMA clones in the 22q11.2 region, but normal copy number detected with the flanking clones (summarized in Fig. 4). The CMA results were independently verified in each case as a duplication by interphase FISH (data not shown). However, because of difficulty determining either the proximal or distal breakpoints in these cases, additional analyses were performed using either FISH or high-resolution oligonucleotide microarray to further characterize the extent of the duplications. Brief case reports are provided below. 
A

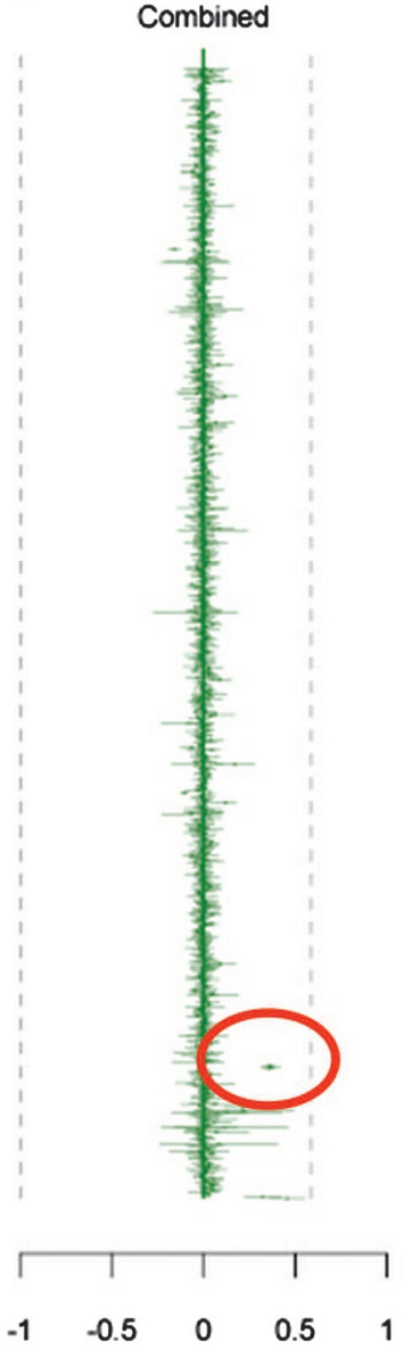

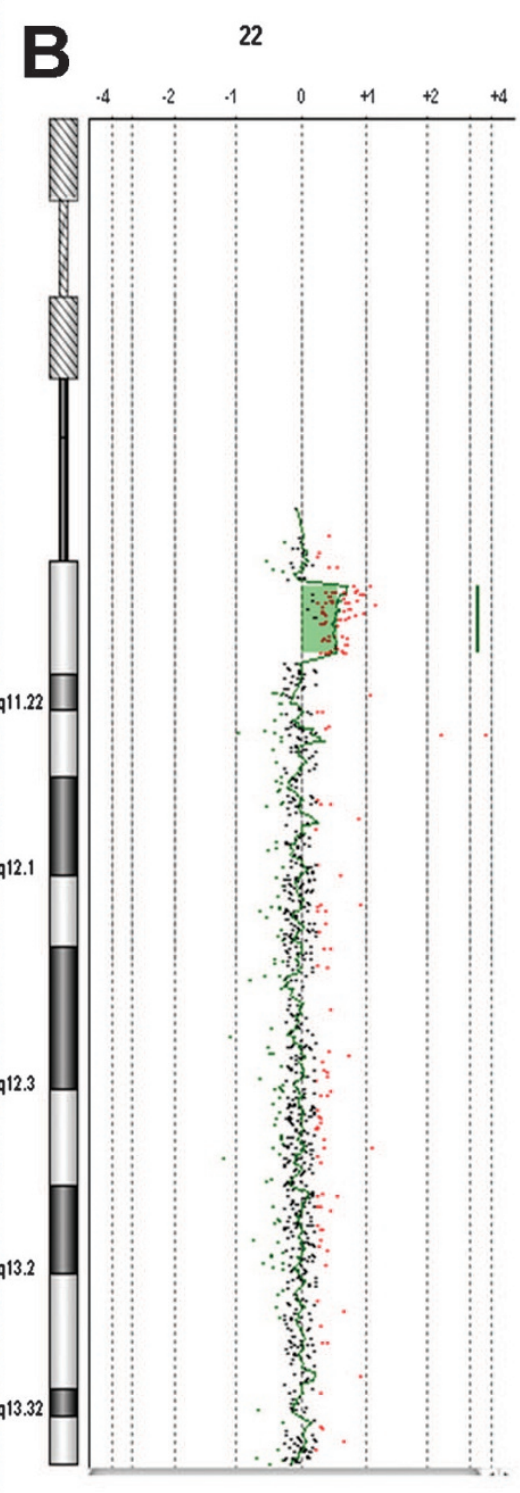
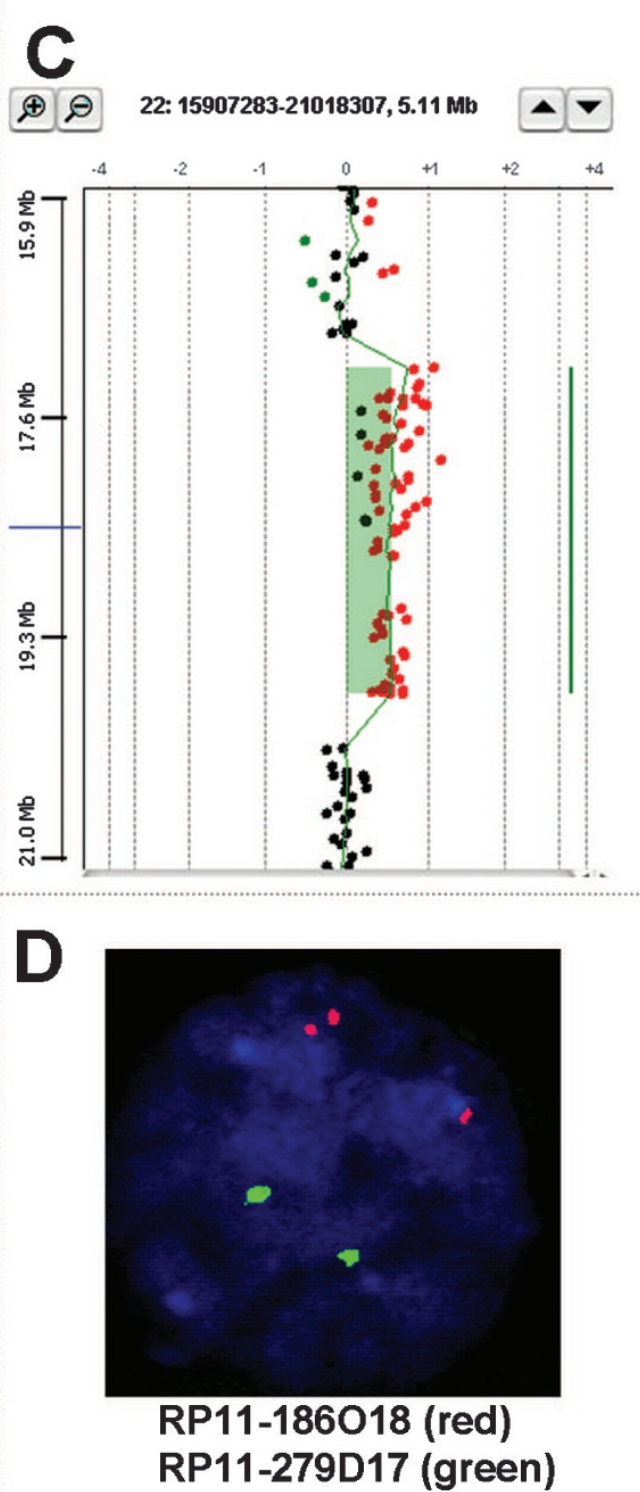

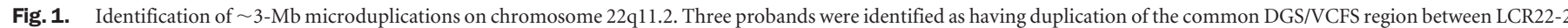

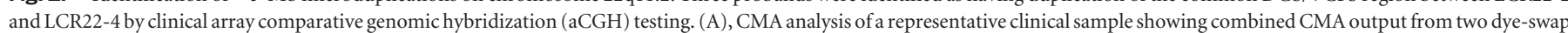

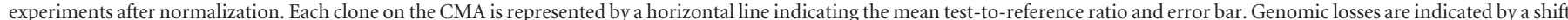

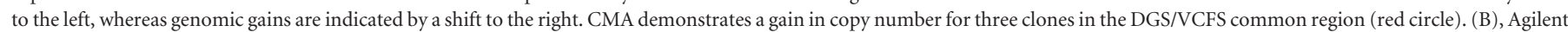

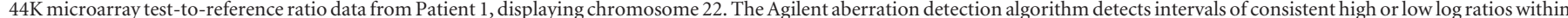

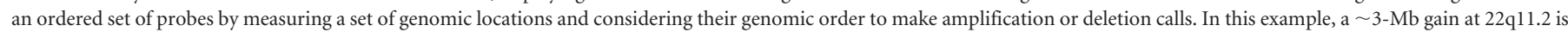

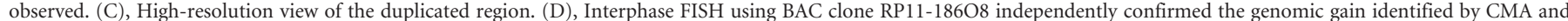
oligoarray as a duplication (three red signals), whereas BAC clone RP11-279D17 located outside the duplicated region showed a normal hybridization pattern (two green signals).

\section{Identification of a novel $\sim 1-\mathrm{Mb}$ "distal nested" $22 q 11.2$ microduplication, LCR22-3a through LCR22-4}

Patient 6 is a 2-month-old infant initially assigned a female gender at birth but later found to have labial masses with sonographic appearance of testes and absence of uterus or ovaries. Urologic evaluation indicated the presence of an underdeveloped phallus with a penoscrotal meatus and severe chordee. Chromosome analysis and FISH for chromosomes $\mathrm{X}$ and $\mathrm{Y}$ revealed a 46,XY chromosome complement and endocrine evaluation revealed a normal male hormonal profile. DNA sequencing of the androgen receptor gene was normal. Based on the anatomic, chromosomal, and hormonal data, the infant was reassigned to a male gender and subsequently underwent orchiopexy. A CMA was obtained as part of the intersex evaluation and confirmed a normal signal for $S R Y$ and revealed gain of copy number with a single clone, RP11-165F18, between LCR 22-3b and LCR 22-4. Flanking clones RP11-316L10 and RP11-36N5 showed normal copy number, indicating an atypical duplication within the distal portion of the DGS/VCFS region. Characterization of the size of the duplication was done by FISH mapping using fosmid clones G248P83456F11 and G248P86496D2 between LCR22-3a and LCR22-3b revealed 
Ou et al.
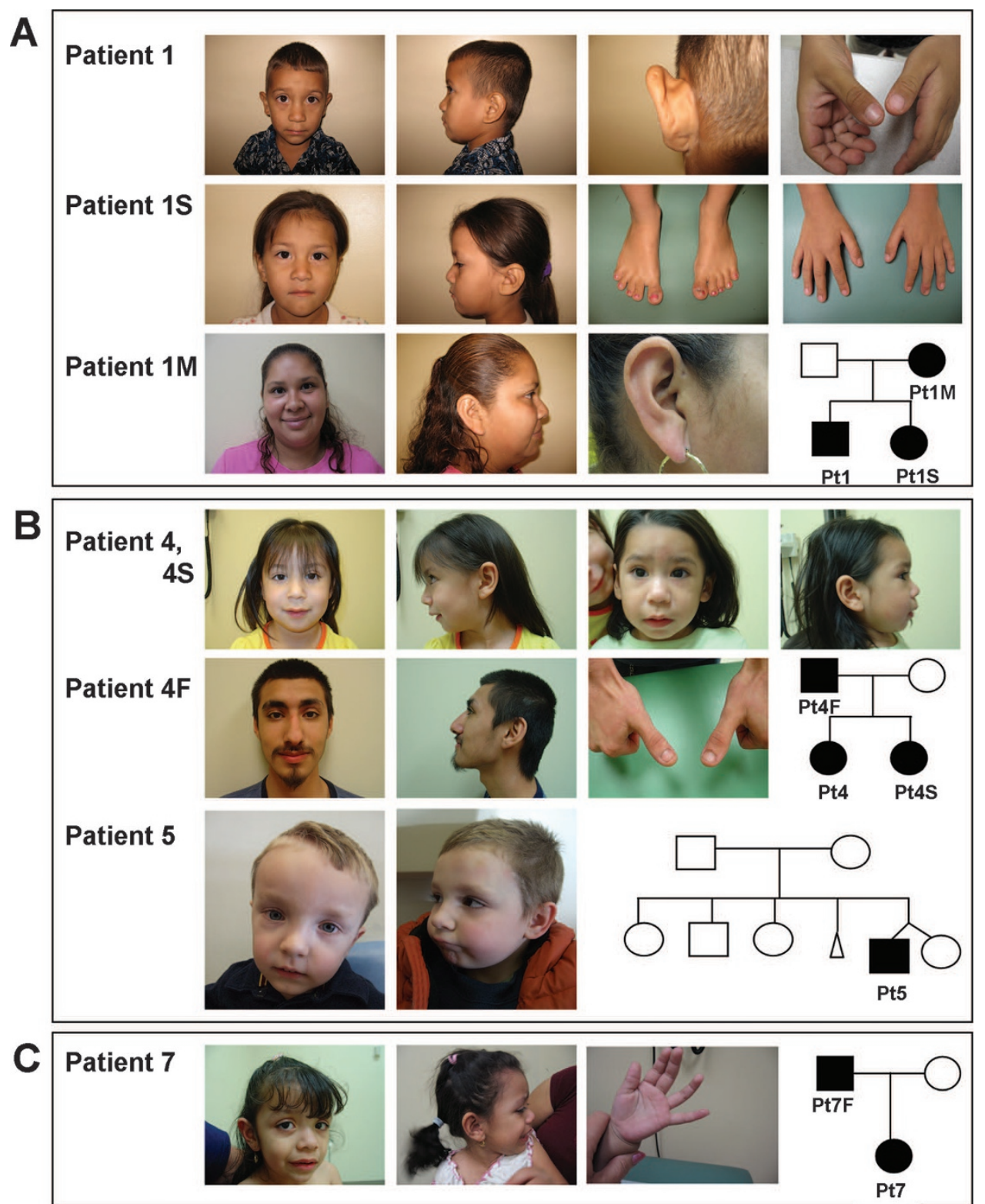

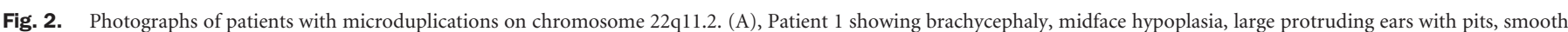

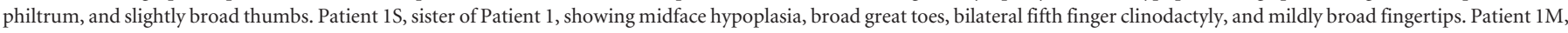

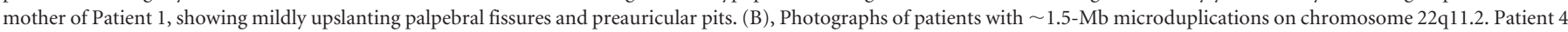

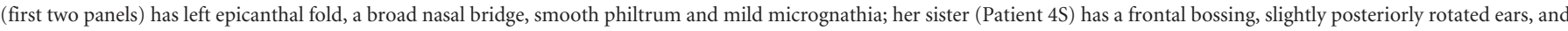

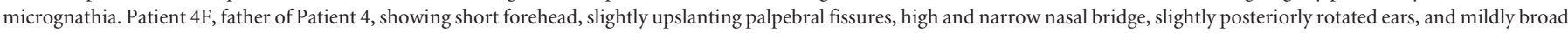

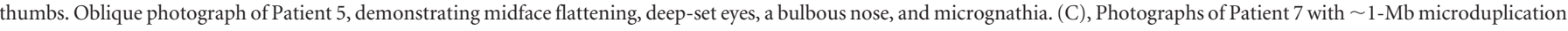

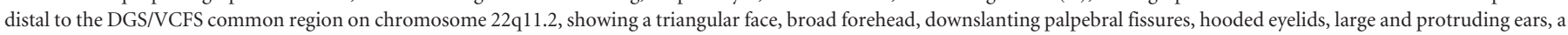
full nasal tip, smooth philtrum, and prominent fingertip pads. Also shown are the pedigrees for each family demonstrating their inheritance.

three signals for each probe, indicating the presence of an atypical 1-Mb microduplication between LCR 22-3a and LCR 22-4 that likely represents the reciprocal recombination product of the atypical deletion reported by Garcia-Miñaur et al. ${ }^{20}$ The father, who is reportedly phenotypically normal, was found to carry the same microduplication by CMA (referred to as Patient 6F).

Identification of a novel $\sim 1-\mathrm{Mb}$ distal $22 q 11.2$ microduplication, LCR22-4 through LCR22-5

Patient 7 is a 28 -month-old female child with developmental delay and dysmorphic features (Fig. 2, C). Developmental milestones included sitting at 7 months, walking at 23 months, and only a few single words by 28 months. On examination at 28 months she had normal growth parameters, a somewhat triangular face, parietal and frontal bossing, broad forehead, an upsweep of the frontal hairline, downslanting palpebral fissures, strabismus, hooded eyelids, large and protruding ears with preauricular pits, a full nasal tip, smooth philtrum, normal palate, short sternum, and prominent fingertip pads. Her evaluation included a normal echocardiogram, electroencephalography, brain magnetic resonance imaging, renal ultrasound, and 


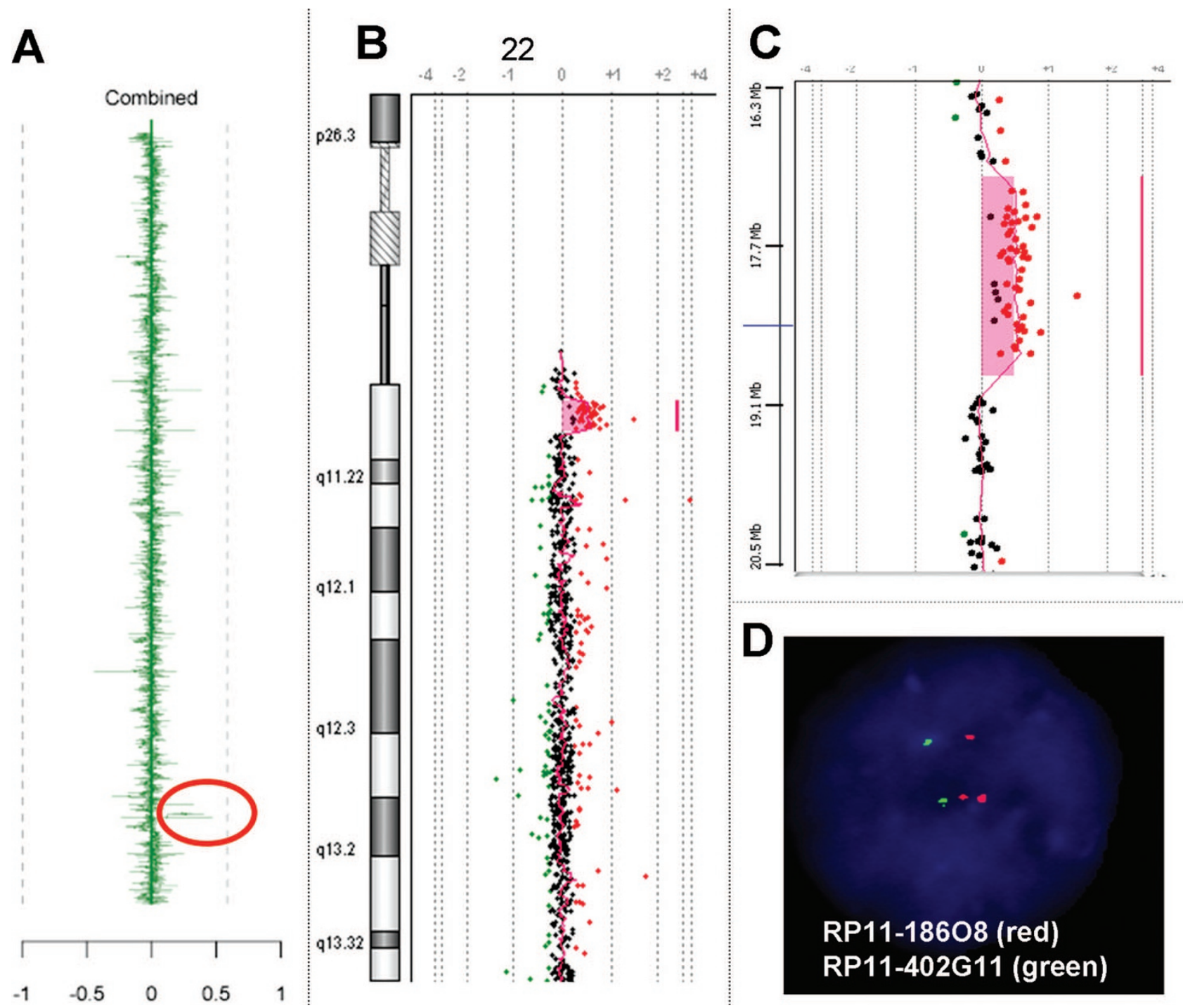

Fig. 3. Identification of $\sim 1.5-\mathrm{Mb}$ proximal "nested" duplications on chromosome 22q11.2. Two probands were identified as having duplication of the proximal nested DGS/VCFS region between LCR22-2 and LCR22-3a by clinical array comparative genomic hybridization (aCGH) testing. (A), CMA analysis of a representative clinical sample showing combined CMA output from two dye-swap experiments after normalization (as in Fig. 1). CMA demonstrates a gain in copy number for two clones in the DGS/VCFS common region (red circle). (B), Agilent $44 \mathrm{~K}$ microarray test-to-reference ratio data for the chromosome 22 demonstrates a $\sim 1.5-\mathrm{Mb}$ gain. (C), A high-resolution view of the $22 \mathrm{q} 11.2$ microduplication is shown. (D), Interphase FISH using BAC clone RP11-186O8 independently confirmed the genomic gain identified by CMA and oligoarray as a duplication (three red signals), whereas BAC clone RP11-402G11 located outside the duplicated region showed a normal hybridization pattern (two green signals).

hearing test. She was found to have gain of copy number with a single CMA clone, RP11-36N5, but normal copy number with flanking clones RP11-165F18 (proximal) and RP5-930L11 (distal), which was independently verified by interphase FISH (not shown). The placement of the clones on the CMA permitted delineation of the likely proximal breakpoints within LCR22-4, but the distal breakpoint was less obvious and could have fallen within LCR22-5, LCR22-6, or LCR22-7. We used the Agilent $44 \mathrm{~K}$ oligonucleotide microarray to further define the duplicated region as extending between probes mapping to nucleotides 20,131,604 bp and 21,246,553 bp based on build hg18. These data are most consistent with an atypical $\sim 1-\mathrm{Mb}$ duplication between LCR22-4 and LCR22-5 distal to the common DGS/VCFS region (summarized in Fig. 4). The patient's father was found to carry the same duplication (referred to as Patient 7F) and has hooded eyelids and a low posterior hairline. He was in regular classes in school and reported no learning disabilities.

\section{Identification of a novel $\sim 2-M b$ distal 22q11.2}

microduplication, LCR22-4 through LCR22-6

Patient 8 was referred for a genetics evaluation at 4 days of life because of multiple congenital anomalies, including imperforate anus, small left kidney ( -2 standard deviations) with duplicated collecting system and bilateral vesicoureteral reflux, patent ductus arteriosus, patent foramen ovale, and anomalous right subclavian artery. Physical examination revealed a widely split metopic suture, low anterior hairline, flat nasal 

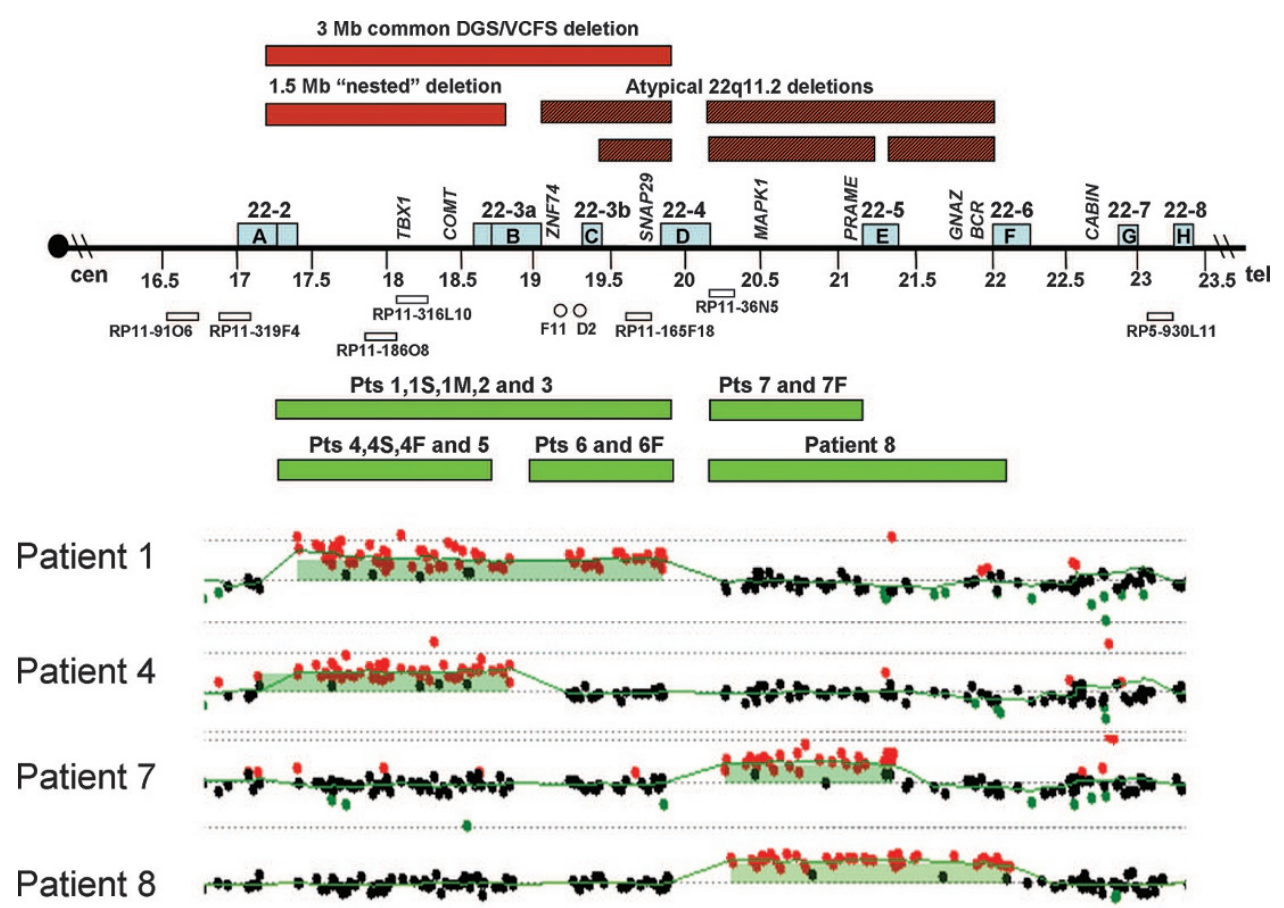

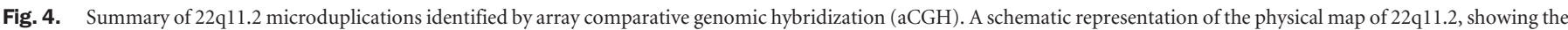

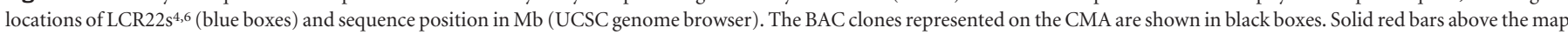

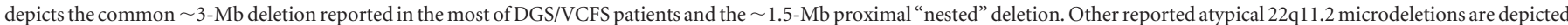

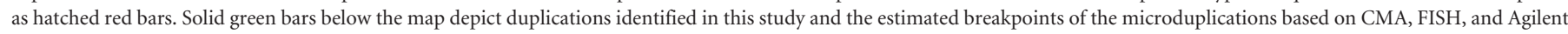

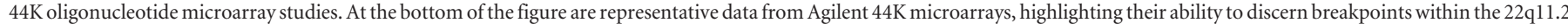
region. Note gaps in oligonucleotide coverage that correspond to LCR regions.

bridge, small downslanting palpebral fissures, normal ears, and normal oropharynx. Both testes were descended, but the left testis was smaller than the right and a skin tag was noted on the scrotum. The extremities showed a triphalangeal thumb on the left and hypoplastic toenails. Neurologic examination was unremarkable. Spine radiographs revealed normal segmentation but possible scoliosis. The family history was positive for a very distant paternal cousin with a rectovaginal fistula. CMA revealed gain of copy number with a single clone, RP11-36N5, but normal copy number with flanking clones RP11-165F18 (proximal) and RP5-930L11, similar to Patient 7. Agilent $44 \mathrm{~K}$ oligonucleotide microarray analysis further define the duplicated region between probes mapping to nucleotides 20,131,604 bp and 21,957,339 bp based on build hg18. These results are most consistent with an atypical duplication of 2-Mb between LCR22-4 and LCR22-6 (summarized in Fig. 4). Parental samples were unavailable at the time of submission.

We inspected whether copy number polymorphism was previously reported for the duplicated regions found in $\mathrm{Pa}$ tients 6-8 (UCSC Genome Browser, Structural Variation track, accessed June 18, 2007). There is no evidence for polymorphism between LCR22-3a and LCR22-4. A duplication of clone RP11-481L22 between LCR22-4 and LCR22-5 was identified in 1 of 269 HapMap individuals, ${ }^{31}$ and copy number polymorphism (both gains and losses) is frequently observed between LCR22-4 and LCR22-6. ${ }^{31-35}$

\section{DISCUSSION}

Reciprocal recombination or strand exchange at flanking LCRs during meiosis was first demonstrated for duplication/deletion syndromes in the region of chromosome 17p12 implicated in the Charcot-Marie-Tooth disease type 1A and hereditary neuropathy with liability to pressure palsies (tomaculous neuropathy). ${ }^{36}$ This mechanism for recurrent rearrangements is also postulated to occur in other genomic disorders and predicts that microdeletions and their reciprocal microduplications should occur with similar frequency. ${ }^{1,10}$ In this investigation, we present results from a clinical aCGH test designed to detect chromosomal abnormalities across the whole genome including 22q11.2. Placement of seven BACs flanking the major LCRs within $22 \mathrm{q} 11.2$ enables this assay to detect a variety of microdeletions and microduplications that occur between the LCR22s in this region. In our experience with 7000 clinical CMA cases, we have detected 40 individuals with deletions in the 22q11.2 region and 19 individuals with duplications, indicating that deletions were twice as common in this particular cohort despite the very broad range of referring indications. One possible explanation for this finding is that the wide phenotypic variability observed in individuals with microduplications of this region (see below) results in less ascertainment of very mildly affected individuals through clinical testing.

We have determined the molecular and clinical characteristics of eight unrelated probands and six family members who 
carry microduplications of chromosome 22q11.2. Among these individuals, three probands harbor the previously reported $\sim 3-\mathrm{Mb}$ microduplication complementary to the common $\sim 3-\mathrm{Mb}$ microdeletion that causes DGS/VCFS. In addition, we have identified individuals with novel microduplications complementary to the proximal nested $\sim 1.5-\mathrm{Mb}$ deletion and distal nested $\sim 1-\mathrm{Mb}$ deletion within the common DGS/VCFS region, as well as atypical microduplications distal to the common DGS/VCFS region (summarized in Fig. 4). While this manuscript was in preparation, a single patient with a $\sim 1.5 \mathrm{Mb}$ proximal nested microduplication was reported by Alberti et al. ${ }^{37}$ and a patient mosaic for cell lines containing the reciprocal $\sim 1.5-\mathrm{Mb}$ microdeletion and microduplication of this region was reported by Dempsey et al., 38 but to our knowledge, the other atypical duplications have not previously been reported in the literature. The CMA results and high-resolution oligonucleotide array results are consistent with breakpoints of these various rearrangements involving most of the LCR22s that occur in this region. Importantly, in patients for whom parental samples were available we observed a high frequency of familial duplications (two of the five probands with common $\sim 3-\mathrm{Mb}$ and proximal "nested" $\sim 1.5-\mathrm{Mb}$ duplications, and both of the probands with smaller distal atypical duplications for whom parental samples were available), consistent with what has been observed by others. ${ }^{9,23,24}$ These results emphasize the importance of investigating other family members for the microduplication.

Overall, there is a wide range of clinical manifestations in patients with microduplication 22q11.2 syndrome, which we have defined here as referring only to those patients with the common $\sim 3-\mathrm{Mb}$ microduplication or the proximal nested $\sim 1.5-\mathrm{Mb}$ microduplication (Tables 1 and 2). Among the more common dysmorphic features were abnormalities of head size and shape, unusual hair patterns, midface flattening, large ears, auricular pits, variable nasal features, smooth philtrum, and digit abnormalities such as squared fingertips and fifth finger clinodactyly. Velopharyngeal insufficiency or hypernasal speech was also noted in several individuals. One patient with a $\sim 3$-Mb duplication (Patient 2 ) was reported to have hypocalcemia in the newborn period but no evidence for abnormal calcium regulation was found in other patients. The degree of developmental delay varied from mild global developmental delay to isolated speech delay or normal cognition. Taken together, the spectrum of features seen in our cohort of patients with microduplication 22q11.2, LCR22-2 through LCR22-4 and microduplication 22q11.2, LCR22-2 through LCR22-3a are somewhat variable and generally milder than those previously reported. There is some degree of overlap with features seen in VCFS/DGS but to a lesser extent than the first reports of 22q11.2 microduplications indicated, which were limited by ascertainment bias. ${ }^{9,23,24}$ Our study avoids this bias to the extent that clinical samples were received for a variety of indications (including developmental delay, dysmorphic features, congenital anomalies, autism, and others), although it is not a truly population-based survey. Four of the five probands with $\sim 3-\mathrm{Mb}$ and $\sim 1.5-\mathrm{Mb}$ proximal nested microduplications in our cohort were referred for testing because of developmental or speech delay and dysmorphic features. One of these individuals (Patient 4) was first tested for DGS/VCFS based on her clinical features, although it should be noted that her microduplication was not initially identified by FISH. Similar findings were reported by Yobb et al. ${ }^{39}$ who found significant variability among patients with $\sim 3-\mathrm{Mb}$ microduplications, with a spectrum of phenotypes, including developmental and speech delay, hearing impairment, failure to thrive, hypotonia, and behavioral abnormalities. The patient with a $\sim 1.5-\mathrm{Mb}$ proximal nested duplication reported by Alberti et al. ${ }^{37}$ has clinical manifestations similar to those observed in the four patients described herein, including developmental delay and dysmorphic features such as narrow forehead, wide nasal bridge, epicanthic folds, upslanting palpebral fissures, and micrognathia. Also, two groups reported finding no individuals with microduplications of 22q11.2 among 372 patients $^{40}$ and 295 patients ${ }^{41}$ referred for clinical testing because of manifestations consistent with DGS/VCFS, suggesting that the microduplication is only rarely associated with this phenotype. In summary, patients with microduplications of 22q11.2 can have a variable phenotype that makes diagnosis based solely on clinical features quite challenging.

Additionally, testing of family members resulted in the ascertainment of several other affected individuals. Familial transmission was found in two of five probands with the common $\sim 3-\mathrm{Mb}$ or $\sim 1.5-\mathrm{Mb}$ proximal nested microduplications (parental samples were not available for two probands).

This rate is similar to that of previous reports. ${ }^{9,39}$ In general, there seems to be variable expressivity between family members carrying the common $\sim 3-\mathrm{Mb}$ and $\sim 1.5-\mathrm{Mb}$ microduplications (to the extent that transmitting parents escaped clinical detection before their child's diagnosis), but virtually all of the individuals carrying these duplications had some degree of developmental delay. A recent report of an antenatal case of 22q11.2 duplication associated with severe cardiovascular anomalies, including total anomalous pulmonary venous return and heterotaxy, inherited from a father with mild cognitive deficits ${ }^{42}$ highlights the potential for extreme variability of phenotypes seen in patients with 22q11.2 duplications even within a family.

Finally, the limited numbers of patients with atypical or distal duplications and the presence of these duplications in phenotypically normal parents restrict our ability to make generalized statements regarding the potential phenotypic consequences of these small rearrangements. Together, these issues highlight the difficulty encountered in genetic counseling and should prompt some degree of caution in interpreting the clinical significance of certain genomic rearrangements, especially given the recent recognition of widespread segmental duplications and copy number variation in the human genome. ${ }^{31-35}$

Until much more information is available regarding individuals with smaller duplications in the 22q11.2 region, perhaps we should consider the microduplication 22q11.2 syndrome to comprise only those individuals with common $\sim 3-\mathrm{Mb}$ or $\sim 1.5-\mathrm{Mb}$ proximal nested microduplications. Given the com- 
plexity of the genomic structure in the region with numerous LCRs capable of mediating NAHR, we propose using expanded nomenclature that clearly identifies the region that is deleted or duplicated, similar to what has been proposed for Williams-Beuren syndrome. ${ }^{43}$ For example, the common duplication could be formally referred to as "microduplication 22q11.2, LCR22-2 through LCR22-4" and the proximal nested $\sim 1.5$-Mb microduplication could be termed "microduplication 22q11.2, LCR22-2 through LCR22-3a.” A limitation to this approach is that the molecular characterization of patients differs greatly between reports and the breakpoints are not always defined. However, detailed molecular characterization and documentation in the literature is critical to making accurate genotype-phenotype correlations. ${ }^{44}$ Use of well-curated databases such as DECIPHER and the Database of Genomic Variants will also improve attempts at genotype-phenotype correlation for rare genomic rearrangements.

The 3-Mb intervals between LCR22-2 to LCR22-4 contain more than 40 genes. ${ }^{45}$ The TBX1 gene, which maps between LCR22-2 and LCR22-3a, is a member of the T-box family of transcription factors, and has been shown to be the major disease gene in this region responsible for the DGS/VCFS. ${ }^{46-49}$ Interestingly, it has been observed in mouse models that both over- and under-expression of TBX1 recapitulates the DGS/ VCFS phenotype. ${ }^{50,51}$ Recently, gain of function mutations in $T B X 1$ have been reported patients with overlapping phenotypes with DGS/VCFS. ${ }^{49,52}$ The above studies suggest that TBX1 dosage outside of normal range may affect the same developmental pathways in humans and contribute to the DGS/ VCFS and the 22q11.2 duplication syndrome. Therefore, altered TBX1 expression together with other additional genetic, epigenetic, or nongenetic factors required for full expressivity may account for the highly variable phenotype seen in patients with microduplications of 22q11.2 that include the TBX1 gene. Additionally, if some of the malformations associated with DGS/VCFS are related to developmental field effects, the pathogenic effect of a deletion could be more severe than that of a duplication. Furthermore, in each of these cases it is distinctly possible that the penetrance of a functional SNP on the nonrearranged chromosome could be affected by the presence of a genomic rearrangement, as has been shown for Sotos syndrome with Factor XII deficiency. ${ }^{53}$

In the course of this study we have also identified several individuals with atypical microduplications in the 22 q11.2 region, and it should be stressed that it remains unclear whether the duplications are associated causally with the phenotypes observed in these individuals.

Patient 6 , who has a novel $\sim 1-\mathrm{Mb}$ microduplication between LCR22-3a and LCR22-4, had severely undervirilized external genitalia with hypospadias and cryptorchidism that led to initial mistaken gender assignment at birth. An investigation for causes of intersex conditions is ongoing and, as noted above, no mutations were identified in the androgen receptor gene. This is the only report of ambiguous genitalia in a patient with microduplication of 22q11.2, although other patients have been noted to have a variety of urogenital abnormalities, including urethral stenosis, mild hypospadias, and hydronephrosis ${ }^{9}$ and cryptorchidism. ${ }^{24}$ Cryptorchidism and hypospadias also occur in patients with chromosome 22q11.2 deletions ${ }^{54}$ along with other malformations of the renal system. It is thus plausible that a dosage-sensitive gene within this interval could contribute to developmental disorders of the genitourinary tract, albeit with reduced penetrance because only a subset of patients with copy number abnormalities of chromosome 22q11.2 show evidence of genitourinary malformations. However, because the patient's phenotypically normal father carries the same microduplication, it is uncertain whether the microduplication should be implicated as the cause of the ambiguous genitalia in this patient.

Patient 7 , who has a novel $\sim 1-\mathrm{Mb}$ microduplication between LCR22-4 and LCR22-5, has developmental delay and dysmorphic features, whereas her father who carries the same microduplication reports no cognitive delay. Patient 8 , who has a novel microduplication (LCR22-4 to LCR22-6) that overlaps with Patient 7 had multiple congenital abnormalities. There are about 21 annotated genes between LCR22-4 to LCR22-6. Many of the genes in this genomic region have not been extensively studied, and therefore, whether the gain of copy number for this region is causative for their phenotype is unclear. Certainly, the identification of more patients with these atypical microduplications will be required for further delineation of the phenotypes associated with these rearrangements.

In conclusion, we have found that microduplications of chromosome 22q11.2 can have diverse molecular and phenotypic manifestations and that these rearrangements are frequently inherited. The phenotype does not strongly overlap with DGS/VCFS, indicating that initial reports may represent patients at the more severe end of the clinical spectrum for this condition. CMA is an ideal modality for clinical testing as it offers greater sensitivity, better delineation of the size and extent of genomic gains and losses, high throughput, and a lesslabor-intensive approach than interphase FISH analysis to screen for a variety of genomic imbalances, including microduplications of chromosome 22q11.2.

\section{WEB RESOURCES}

The URLs for data presented herein are as follows:

BCM Medical Genetics Laboratories Website, http:// www.bcm.edu/cma/assets/abnormalities.pdf

Online Mendelian Inheritance in Man (OMIM), http://www.ncbi.nlm.nih.gov/Omim/ (for DGS and VCFS)

UCSC Genome Browser, Structural Variation track, http://genome.ucsc.edu/cgi-bin/hgTracks

DECIPHER, http://www.sanger.ac.uk/PostGenomics/ decipher/

Database of Genomic Variants, http://projects.tcag.ca/ variation/

\section{ACKNOWLEDGMENTS}

We thank all the families in this study for their participation. 


\section{References}

1. Lupski JR. Genomic disorders: structural features of the genome can lead to DNA rearrangements and human disease traits. Trends Genet 1998;14:417-422.

2. Stankiewicz P, Lupski JR. Genome architecture, rearrangements and genomic disorders. Trends Genet 2002;18:74-82.

3. Edelmann L, Pandita RK, Spiteri E, Funke B, et al. A common molecular basis for rearrangement disorders on chromosome 22q11. Hum Mol Genet 1999;8:11571167.

4. Shaikh TH, O'Connor RJ, Pierpont ME, McGrath J, et al. Low copy repeats mediate distal chromosome 22q11.2 deletions: sequence analysis predicts breakpoint mechanisms. Genome Res 2007;17:482-491.

5. McDermid HE, Morrow BE. Genomic disorders on 22q11. Am J Hum Genet 2002; 70:1077-1088.

6. Edelmann L, Pandita RK, Morrow BE. Low-copy repeats mediate the common 3-Mb deletion in patients with velo-cardio-facial syndrome. Am J Hum Genet 1999; 64:1076-1086.

7. McDermid HE, Duncan AM, Brasch KR, Holden JJ, et al. Characterization of the supernumerary chromosome in cat eye syndrome. Science 1986;232:646-648.

8. Zackai EH, Emanuel BS. Site-specific reciprocal translocation, t(11;22) (q23; q11), in several unrelated families with 3:1 meiotic disjunction. Am J Med Genet 1980;7:507-521.

9. Ensenauer RE, Adeyinka A, Flynn HC, Michels VV, et al. Microduplication 22q11.2, an emerging syndrome: clinical, cytogenetic, and molecular analysis of thirteen patients. Am J Hum Genet 2003;73:1027-1040.

10. Shaffer LG, Lupski JR. Molecular mechanisms for constitutional chromosomal rearrangements in humans. Annu Rev Genet 2000;34:297-329.

11. Shprintzen RJ, Goldberg RB, Young D, Wolford L. The velo-cardio-facial syndrome: a clinical and genetic analysis. Pediatrics 1981;67:167-172.

12. Scambler PJ, Kelly D, Lindsay E, Williamson R, et al. Velo-cardio-facial syndrome associated with chromosome 22 deletions encompassing the DiGeorge locus. Lancet 1992;339:1138-1139.

13. McDonald-McGinn DM, Tonnesen MK, Laufer-Cahana A, Finucane B, et al. Phenotype of the 22q11.2 deletion in individuals identified through an affected relative: cast a wide FISHing net! Genet Med 2001;3:23-29.

14. Carlson C, Sirotkin H, Pandita R, Goldberg R, et al. Molecular definition of 22q11 deletions in 151 velo-cardio-facial syndrome patients. Am J Hum Genet 1997;61: $620-629$.

15. Rauch A, Pfeiffer RA, Leipold G, Singer H, et al. A novel 22q11.2 microdeletion in DiGeorge syndrome. Am J Hum Genet 1999;64:659-666.

16. Kurahashi H, Nakayama T, Osugi Y, Tsuda E, et al. Deletion mapping of 22q11 in CATCH22 syndrome: identification of a second critical region. Am J Hum Genet 1996;58:1377-1381.

17. Amati F, Conti E, Novelli A, Bengala M, et al. Atypical deletions suggest five 22q11.2 critical regions related to the DiGeorge/velo-cardio-facial syndrome. Eur J Hum Genet 1999;7:903-909.

18. McQuade L, Christodoulou J, Budarf M, Sachdev R, et al. Patient with a 22q11.2 deletion with no overlap of the minimal DiGeorge syndrome critical region (MDGCR). Am J Med Genet 1999;86:27-33.

19. Saitta SC, McGrath JM, Mensch H, Shaikh TH, et al. A 22q11.2 deletion that excludes UFD1L and CDC45L in a patient with conotruncal and craniofacial defects. Am J Hum Genet 1999;65:562-566.

20. Garcia-Miñaur S, Fantes J, Murray RS, Porteous MEM, et al. A novel atypical 22q11.2 distal deletion in father and son. J Med Genet 2002;39:E62.

21. Rauch A, Zink S, Zweier C, Thiel CT, et al. Systematic assessment of atypical deletions reveals genotype-phenotype correlation in 22q11.2. J Med Genet 2005;42:871876.

22. Ben-Shachar S, Ou Z, Shaw CA, Belmont JW, et al. 22q11.2 distal deletion: a novel recurrent genomic disorder distinct from DiGeorge syndrome/velocardiofacial syndrome. Am J Hum Genet 2008;82:214-221.

23. Hassed SJ, Hopcus-Niccum D, Zhang L, Li S, et al. A new genomic duplication syndrome complementary to the velocardiofacial (22q11 deletion) syndrome. Clin Genet 2004;65:400 - 404

24. Portnoï MF, Lebas F, Gruchy N, Ardalan A, et al. 22q11.2 duplication syndrome: two new familial cases with some overlapping features with DiGeorge/velocardiofacial syndromes. Am J Med Genet A 2005;137:47-51.

25. Sparkes R, Chernos J, Dicke F. Duplication of the 22q11.2 region associated with congenital cardiac disease. Cardiol Young 2005;15:229-231.

26. Cheung SW, Shaw CA, Yu W, Li J, et al. Development and validation of a CGH microarray for clinical cytogenetic diagnosis. Genet Med 2005;7:422-432.

27. Stankiewicz P, Beaudet AL. Use of array CGH in the evaluation of dysmorphology, malformations, developmental delay, and idiopathic mental retardation. Curr Opin Genet Dev 2007;17:182-192.
28. Cai WW, Mao JH, Chow CW, Damani S, et al. Genome-wide detection of chromosomal imbalances in tumors using BAC microarrays. Nat Biotechnol 2002;20:393396.

29. Lu X, Shaw CA, Patel A, Li J, et al. Clinical implementation of chromosomal microarray analysis: summary of 2513 postnatal cases. PLoS ONE 2007;2:e327.

30. Probst FJ, Roeder ER, Enciso VB, Ou Z, et al. Chromosomal microarray analysis (CMA) detects a large X chromosome deletion including FMR1, FMR2, and IDS in a female patient with mental retardation. Am J Med Genet A 2007;143:1358 1365 .

31. Locke DP, Sharp AJ, McCarroll SA, McGrath SD, et al. Linkage disequilibrium and heritability of copy-number polymorphisms within duplicated regions of the human genome. Am J Hum Genet 2006;79:275-290.

32. Sharp AJ, Locke DP, McGrath SD, Cheng Z, et al. Segmental duplications and copy-number variation in the human genome. Am J Hum Genet 2005;77:78-88.

33. Redon R, Ishikawa S, Fitch KR, Feuk L, et al. Global variation in copy number in the human genome. Nature 2006;444:444-454.

34. Sebat J, Lakshmi B, Troge J, Alexander J, et al. Large-scale copy number polymor phism in the human genome. Science 2004;305:525-528.

35. McCarroll SA, Hadnott TN, Perry GH, Sabeti PC, et al. Common deletion polymorphisms in the human genome. Nat Genet 2006;38:86-92.

36. Chance PF, Abbas N, Lensch MW, Pentao L, et al. Two autosomal dominant neuropathies result from reciprocal DNA duplication/deletion of a region on chromosome 17. Hum Mol Genet 1994;3:223-228.

37. Alberti A, Romano C, Falco M, Cali F, et al. $1.5 \mathrm{Mb}$ de novo 22q11.21 microduplication in a patient with cognitive deficits and dysmorphic facial features. Clin Genet 2007;71:177-182.

38. Dempsey MA, Schwartz S, Waggoner DJ. Mosaicism del(22)(q11.2q11.2)/ $\operatorname{dup}(22)(\mathrm{q} 11.2 \mathrm{q} 11.2)$ in a patient with features of 22q11.2 deletion syndrome. $A m \mathrm{~J}$ Med Genet A 2007;143:1082-1086.

39. Yobb TM, Somerville MJ, Willatt L, Firth HV, et al. Microduplication and triplication of 22q11.2: a highly variable syndrome. Am J Hum Genet 2005;76:865-876.

40. Cotter PD, Nguyen H, Tung G, Rauen KA. Incidence of microduplication 22q11.2 in patients referred for FISH testing for velo cardiofacial and DiGeorge syndromes. Eur J Hum Genet 2005;13:1245-1246.

41. Brunet A, Gabau E, Perich RM, Valdesoiro L, et al. Microdeletion and microduplication 22q11.2 screening in 295 patients with clinical features of DiGeorge/velocardiofacial syndrome. Am J Med Genet A 2006;140:2426-2432.

42. de La Rochebrochard C, Joly-Hélas G, Goldenberg A, Durand I, et al. The intrafamilial variability of the $22 \mathrm{q} 11.2$ microduplication encompasses a spectrum from minor cognitive deficits to severe congenital anomalies. Am J Med Genet A 2006;140: $1608-1613$.

43. Morris CA, Mervis CB, Hobart HH, Gregg RG, et al. GTF2I hemizygosity implicated in mental retardation in Williams syndrome: genotype-phenotype analysis of five families with deletions in the Williams syndrome region. Am J Med Genet A 2003; 123:45-59.

44. Biesecker LG. Lumping and splitting: molecular biology in the genetics clinic. Clin Genet 1998;53:3-7.

45. Paylor R, Lindsay E. Mouse models of 22q11 deletion syndrome. Biol Psychiatry 2006;59:1172-1179.

46. Jerome LA, Papaioannou VE. DiGeorge syndrome phenotype in mice mutant for the T-box gene, Tbx1. Nat Genet 2001;27:286-291.

47. Merscher S, Funke B, Epstein JA, Heyer J, et al. TBX1 is responsible for cardiovascular defects in velo-cardio-facial/DiGeorge syndrome. Cell 2001;104:619-629.

48. Lindsay EA, Vitelli F, Su H, Morishima M, et al. Tbx1 haploinsufficiency in the DiGeorge syndrome region causes aortic arch defects in mice. Nature 2001;410:97-101.

49. Yagi H, Furutani Y, Hamada H, Sasaki T, et al. Role of TBX1 in human del22q11.2 syndrome. Lancet 2003;362:1366-1373.

50. Funke B, Epstein JA, Kochilas LK, Lu MM, et al. Mice overexpressing genes from the 22q11 region deleted in velo-cardio-facial syndrome/DiGeorge syndrome have middle and inner ear defects. Hum Mol Genet 2001;10:2549-2556.

51. Liao J, Kochilas L, Nowotschin S, Arnold JS, et al. Full spectrum of malformations in velo-cardio-facial syndrome/DiGeorge syndrome mouse models by altering Tbx 1 dosage. Hum Mol Genet 2004;13:1577-1585.

52. Zweier C, Sticht H, Aydin-Yaylagül I, Campbell CE, et al. Human TBX1 missense mutations cause gain of function resulting in the same phenotype as 22q11.2 deletions. Am J Hum Genet 2007;80:510 -517.

53. Kurotaki N, Shen JJ, Touyama M, Kondoh T, et al. Phenotypic consequences of genetic variation at hemizygous alleles: Sotos syndrome is a contiguous gene syndrome incorporating coagulation factor twelve (FXII) deficiency. Genet Med 2005;7:479-483.

54. Wu HY, Rusnack SL, Bellah RD, Plachter N, et al. Genitourinary malformations in chromosome 22q11.2 deletion. J Urol 2002;168:2564-2565. 